\title{
Simulating laser pulse propagation and low-frequency wave emission in capillary plasma channel systems with a ponderomotive guiding center model
}

\author{
Peter Messmer* and David L. Bruhwiler \\ Tech-X Corporation, Boulder, Colorado 80303, USA \\ (Received 17 January 2006; published 13 March 2006)
}

\begin{abstract}
Capillary channels of $\approx 3 \mathrm{~cm}$ in length and with plasma densities $\approx 10^{18} \mathrm{~cm}^{-3}$ are a promising alternative to the much shorter, higher-density gas jets for $\mathrm{GeV}$-scale laser wakefield acceleration of electrons. However, the large discrepancy between length scales of the plasma and the laser presents a major computational challenge for particle-in-cell (PIC) simulations. Methods are therefore sought that relax the need to concurrently resolve both length scales. For example, the commonly used "moving window" algorithm enables a reduction of the computational domain to a few plasma wavelengths, which is orders of magnitude smaller than the full length of the laser-plasma interaction. In addition, ponderomotive guiding center methods enable relaxation of the constraint to resolve the laser wavelength. These averaging methods split the laser-induced current into a rapidly varying part and a slowly varying envelope. The average over fast time scales is performed in a semianalytic way, leaving the evolution of the laser envelope and the plasma response to be modeled numerically. Here, we present a ponderomotive guiding center algorithm and demonstrate its applicability to model capillary channels by comparing it with fully kinetic PIC simulations.
\end{abstract}

DOI: 10.1103/PhysRevSTAB.9.031302

PACS numbers: $52.38 . \mathrm{Kd}$

\section{INTRODUCTION}

The maximum achievable accelerating gradient is orders of magnitude larger when sustained by the collective fields of a high-density plasma, rather than an evacuated metal structure. This has been clearly demonstrated for electrons in laser wakefield accelerator (LWFA) experiments [1-3]. Plasma-based electron acceleration concepts (see [4] for a review) can sustain longitudinal electric fields of the order of the nonrelativistic wave breaking field, $E_{0}=\mathrm{cm}_{e} \omega_{p} / e$, where $\omega_{p}^{2}=4 \pi n_{e} e^{2} / m^{2}$ is the plasma frequency at an electron density $n_{e}$. For $n_{e}=10^{18} \mathrm{~cm}^{-3}$, the electric field is $E_{0} \approx 100 \mathrm{GV} / \mathrm{m}$, with a phase velocity close to the speed of light.

Laser pulse propagation in vacuum is limited by diffraction to the Rayleigh length, $z_{0}=\pi w_{0}^{2} / \lambda$, where $w_{0}$ is the waist size and $\lambda$ the wavelength. In order to sustain the interaction between these strong wakefields and the accelerating electrons, the laser pulse must be guided over many Rayleigh lengths (see e.g. [5] and references therein).

For example, the well-defined electron beams seen in [1] were obtained using a plasma channel to guide a laser pulse of lower energy compared to those in [2,3], in which plasma channel guiding was not used.

Ongoing work in the L'OASIS Program at Lawrence Berkeley National Laboratory, directed toward the production of $\mathrm{GeV}$ electron beams from an LWFA, now includes the propagation of $\approx 100 \mathrm{TW}$ laser pulses down $3 \mathrm{~cm}$ long plasma channels, generated from $\approx 10^{18} \mathrm{~cm}^{-3}$ hydrogen gas in a capillary discharge [6]. For such plasma densities (and lower), the use of Ti-sapphire laser technology with

*Electronic address: messmer@txcorp.com $\lambda=0.8 \mu \mathrm{m}$ leads to a very high ratio of $\omega / \omega_{p}$, making it computationally challenging to simultaneously resolve the small laser wavelength, while capturing the larger spatial scales and long propagation times. For these reasons, a ponderomotive treatment of the laser pulse is essential for rapidly modeling LWFA experiments such as those now underway at LBNL.

The orders of magnitude shorter run times for a ponderomotive model, as compared to a fully kinetic simulation, enable parameter scans and system optimization. Likewise, one can explore the use of coherent transition radiation (CTR) as a diagnostic to measure the electron bunch length as it exits the plasma. Given its low frequency, and dependence on the transverse size of the plasma, CTR is dramatically easier to simulate when one does not have to resolve the laser frequency and wavelength.

The paper is organized in the following manner: Section II describes the envelope model implemented in VORPAL, with particular emphasis on the envelope field solver. In Sec. III we present some benchmark tests of field solver, both for the vacuum case and in the presence of a plasma. The emission of CTR is considered in Sec. IV and we conclude in Sec. V.

\section{ENVELOPE MODEL}

The ponderomotive guiding center model used for these investigations is based on the algorithm developed by Gordon et al. [7] and Mora et al. [8]. However, in order to implement the model in the versatile plasma simulation code VORPAL [9], we use a formulation based on fields, rather than potentials. For completeness, we present the model equations here. 
The electromagnetic field is described by the wave equation for the vector potential in the Coulomb gauge,

$$
\begin{aligned}
\left(\Delta-\frac{1}{c^{2}} \partial_{t t}\right)\left(\tilde{a}_{L}+\tilde{a}_{W}\right)= & -\mu_{0}\left(j_{L}+j_{W}\right) \\
& +\frac{1}{c^{2}} \nabla \partial_{t}\left(\phi_{L}+\phi_{W}\right),
\end{aligned}
$$

where $\tilde{a}_{L}, j_{L}$ are the vector potential of the laser and the laser-induced current, and $\tilde{a}_{W}, j_{W}$ are the same quantities for the plasma wake. The electrostatic potential is also decomposed into a component due to the laser pulse, $\phi_{L}$, and of the wake, $\phi_{W}$. The field evolution can therefore be decoupled into a wave equation for the laser field and one for the wake field,

$$
\left(\Delta-\partial_{t t}\right) \tilde{a}_{\{L, W\}}=-j_{\{L, W\}}+\nabla \partial_{t} \phi_{\{L, W\}},
$$

where $L, W$ indicates the laser or the wake, respectively.

The vector potential of the laser pulse can be decomposed into the oscillatory part and a slowly varying complex envelope $a$,

$$
\tilde{a}_{L}=\frac{a}{2} e^{i \phi}+\text { c.c., }
$$

where $\phi=k x-\omega t$ is the wave phase, and $k, \omega$ are the wave number and frequency. We assume linear polarization, so the complex amplitude $a$ is a scalar.

In the Coulomb gauge, the scalar potential satisfies

$$
\nabla \frac{\partial \phi_{L}}{\partial_{t}}=\frac{1}{\epsilon_{0}} j_{L L}=0,
$$

where $j_{L L}$ is the longitudinal part of the laser-induced current. Equation (4) results from neglecting the transverse shape of the laser pulse, in which case the laser-induced current is purely transverse.

The transverse current density induced by the laser, $j_{L T}$ averaged over a laser period, can be approximated as [8]

$$
j_{L T}=-a \sum_{i} \frac{q_{i} \rho_{i}}{\left\langle m_{i}\right\rangle}=-a \chi,
$$

where $q_{i}$ and $\rho_{i}$ are the charge and charge density of the $i$ th particle, and $m_{i}$ is the instantaneous relativistic mass and the angular brackets represent the average over a laser period. The average relativistic mass is given by

$$
\left\langle m_{i}\right\rangle^{2}=m_{0}^{2}+\frac{P_{i}^{2}}{c^{2}}+\frac{q_{i}^{2}|a|^{2}}{2 c^{2}},
$$

where $P_{i}$ is the particle momentum.

The particle dynamics is explicitly followed only on the slow time scales. The rapidly oscillating laser field affects the particles only via the ponderomotive force, yielding the equation of motion [8]

$$
\partial_{t} P_{i}=q_{i}\left(E+v_{i} \times B-\frac{q_{i}}{4 m_{i}} \nabla|a|^{2}\right),
$$

where $v_{i}$ is the particle velocity, while $E$ and $B$ are the slowly varying electric and magnetic wake fields. We have dropped the angular brackets for notational convenience.

When the radiation is dominantly in the forward direction, the evolution of the laser pulse is described by a wave equation of the form $[8,10]$

$$
\left(\Delta_{T}-\frac{2 i \omega}{c^{2}} \partial_{\tau}+\frac{2}{c} \partial_{\tau \xi}\right) a=-\mu_{0} \chi a,
$$

where we have transformed into the frame of reference moving with the laser pulse, $\xi=x-c t$ and $\tau=t$ and $\Delta_{T}$ is the transverse Laplacian. Solving Eq. (8) evolves the laser envelope in the plasma, with the average laserinduced current determined by the charge density and Eq. (5). Equation (7) determines then the dynamics of the particles, which requires knowledge of the wake field. The wake field is determined by Eq. (2), which is equivalent to solving the full set of Maxwell's equations for the plasma response to the laser envelope.

\section{A. Field solver}

Instead of evolving the entire electromagnetic field, the previous section showed that the simulation can advance two separate fields, one for the laser pulse and one for the wake. The laser field is advanced by rewriting Eq. (8) as in [7],

$$
\left(1+\frac{c^{2}}{\omega^{2}} \partial_{\xi}^{2}\right) \partial_{\tau} a=\frac{c^{2}}{2 i \omega}\left(1+\frac{c}{i \omega} \partial_{\xi}\right)\left(\mu_{0} \chi a+\Delta_{T} a\right) .
$$

Neglecting the $O\left(1 / \omega^{2}\right)$ term, the equation can be transformed back into ordinary coordinates via the substitutions $\partial_{\tau}=\partial_{x}+c \partial_{t}$ and $\partial_{\xi}=\partial_{x}$, yielding

$$
\left(\partial_{x}+c \partial_{t}\right) a=\frac{c^{2}}{2 i \omega}\left(1+\frac{c}{i \omega} \partial_{x}\right)\left(\mu_{0} \chi a+\Delta_{T} a\right) .
$$

This results in an approximation to the dispersion relation of the EM wave in a plasma. However, this relation can be made arbitrarily close to the actual dispersion relation by retaining high-order derivatives on the right side of Eq. (10), as is done in $[11,12]$.

Evolution of the envelope, described by Eq. (10), can be solved stably by using a Crank-Nicholson scheme, which results in an implicit equation of the form

$$
\frac{1}{2 \Delta t}\left(a^{t+1}-a^{t-1}\right)=G\left(\Delta_{T} a^{t-1}, \Delta_{T} a^{t+1}, \partial_{x} a^{t}, \chi a^{t}\right),
$$

where $G$ represents the finite difference stencil for Eq. (10). The time step of this method is twice the fundamental time step. This will result in the requirement to advance two envelope fields interchangeably. Equation (11) can be rewritten as a system of complex linear equations of the form

$$
M a^{t+1}=b\left(a^{t-1}, \partial_{x} a^{t}, \chi a^{t}\right)
$$

where the Laplacian operators are approximated by finite 
difference stencils. This set of linear equations can be solved efficiently using standard linear solver techniques.

In order to evolve the wake field, Eq. (2) has to be solved. However, instead of solving the equation for the wake potential, as was done in [7], we solve the full set of Maxwell's equations for the wake fields. This has the advantage that using a charge conserving current deposition algorithm [13] enables use of the highly efficient electromagnetic PIC algorithm to advance the $E$ and $B$ field in time.

\section{B. Update cycle}

The main complication of the ponderomotive guiding center model is to obtain a stable time-centered scheme for the simultaneous advance of the envelope field, the particles and the wake field. Figure 1 shows the relative timing of all the update steps. A time-centered scheme requires two envelope fields, which are advanced alternately.

The update proceeds as follows. First, envelope field $a_{1}$ is updated from time step $n-1 / 2$ to $n+3 / 2$, with a step size of $2 \Delta t$, using a discretized version of Eq. (10). Then,

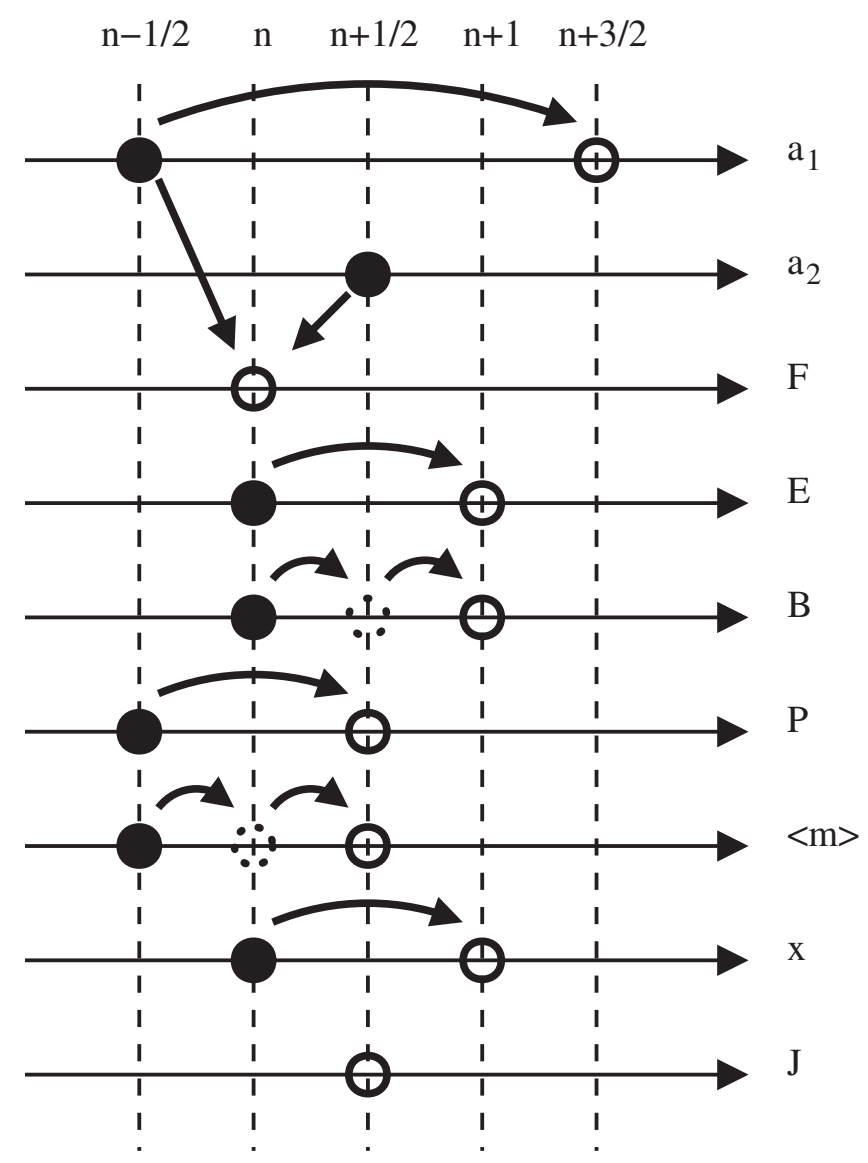

FIG. 1. Time stepping of the envelope field solver, the electromagnetic PIC update and the particle dynamics. Full circles indicate the initial state, dotted circles intermediate steps and empty circles the quantities at the end of the time step. the ponderomotive force is obtained as the average of the two envelope fields, $F^{n}=\left(\nabla\left|a_{1}^{n-1 / 2}\right|+\nabla\left|a_{2}^{n+1 / 2}\right|\right) / 2$.

At the time of the particle position advance in the standard electromagnetic PIC code, both $E$ and $B$ are known. The time derivative of Eq. (6) therefore leads to an implicit equation for the mass at time step $n$,

$$
m^{n}=m^{n-1 / 2}+q\left(E^{n}-\frac{q}{4 m^{n}} F^{n}\right) \frac{P^{n-1 / 2}}{m^{n-1 / 2}} \frac{\Delta t}{2},
$$

which can be solved via the quadratic formula. The next step is to update the momentum via a Boris push [14], with the electric field corrected by the ponderomotive force:

$$
\begin{gathered}
I_{i}=q_{i}\left(E_{i}^{n}-\frac{1}{4} \frac{q_{i}}{m_{i}^{n}} F_{i}^{n}\right), \\
P_{i}^{n+1 / 2}=R\left(P_{i}^{n-1 / 2}+\frac{I_{i}}{2}\right)+\frac{I_{i}}{2},
\end{gathered}
$$

where the operator $R$ effects a rotation of the momentum vector around the local magnetic field through an angle

$$
\Theta=-\frac{q_{i} B^{n}}{m_{i}^{n}} \Delta t
$$

In the next step, the mass is advanced by another half time step, via

$$
\left(m_{i}^{n+1 / 2}\right)^{2}=m_{0}^{2}+\frac{\left(P_{i}^{n+1 / 2}\right)^{2}}{c^{2}}+\frac{q_{i}^{2} a^{2}}{2 c^{2}},
$$

where

$$
a^{2}=\left[a_{1}^{n+1 / 2}\left(r_{i}^{n}\right)\right]^{2}+\nabla\left[a_{1}^{n+1 / 2}\left(r_{i}^{n}\right)\right]^{2} \cdot \frac{P_{i}^{n+1 / 2}}{m_{i}^{n+1 / 2}} \frac{\Delta t}{2} .
$$

Equation (17) is a cubic equation and can be solved numerically using a few iterations of a Newton scheme. With the mass known at time $n+1 / 2$, the conductivity $\chi$ can be determined from Eq. (5). This allows to update the envelope field $a_{1}$ to time step $n+3 / 2$.

Finally, the particle position is updated via

$$
x_{i}^{n+1}=x_{i}^{n}+\frac{P_{i}^{n+1 / 2}}{m_{i}^{n+1 / 2}} \Delta t,
$$

which leads to the determination of the current density via the charge conserving current deposition algorithm. What follows next is a regular Yee update for the wake field.

This completes one update step. The next update step involves the second envelope field, $a_{2}$, but otherwise has the same form. One then repeats these two update steps as often as necessary.

\section{Implementation}

A key problem of the envelope model is how to solve the linear system of complex equations resulting from the discretization of Eq. (10). In order to solve the system, 
we use Krylov subspace solvers from the parallel solver package AZTEC [15]. This has several advantages: (a) an electrostatic solver is already implemented in VORPAL, based on the AZTEC package [16], (b) parallelization of the solver is readily available, and (c) the package allows to experiment with different solvers and preconditioners without time consuming implementation. Using a general purpose solver library can also be beneficial for future work, like enhancing the approximation of Eq. (10).

One problem associated with the AZTEC package is that it only provides solvers for real quantities. In order to solve the complex envelope equation, we therefore solved a system for the real and imaginary parts,

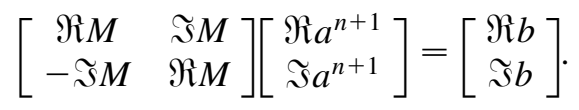

While this results in a slightly more complicated matrix, it results in a significantly simpler solver that can easily be adapted to different envelope evolution equations.

\section{BENCHMARK PROBLEMS}

In order to validate the model, we performed a variety of tests for problems with well-known solutions. We split these tests into tests of the pure field solver, field solver in comparison with a plasma and finally cross checks with full PIC simulations.

\section{A. Vacuum propagation}

A first benchmark to test the envelope model is to investigate laser pulse propagation in vacuum. Figure 2 shows amplitude contours for a laser pulse launched at $z=$ $-2 z_{0}$, where $z_{0}=k w_{0}^{2} / 2$ is the Rayleigh length, $k$ the laser pulse wave number, and $w_{0}$ the pulse width. The size of the computational domain is $12 z_{0} \times 20 w_{0}$, discretized on a $70 \times 60$ cell grid.

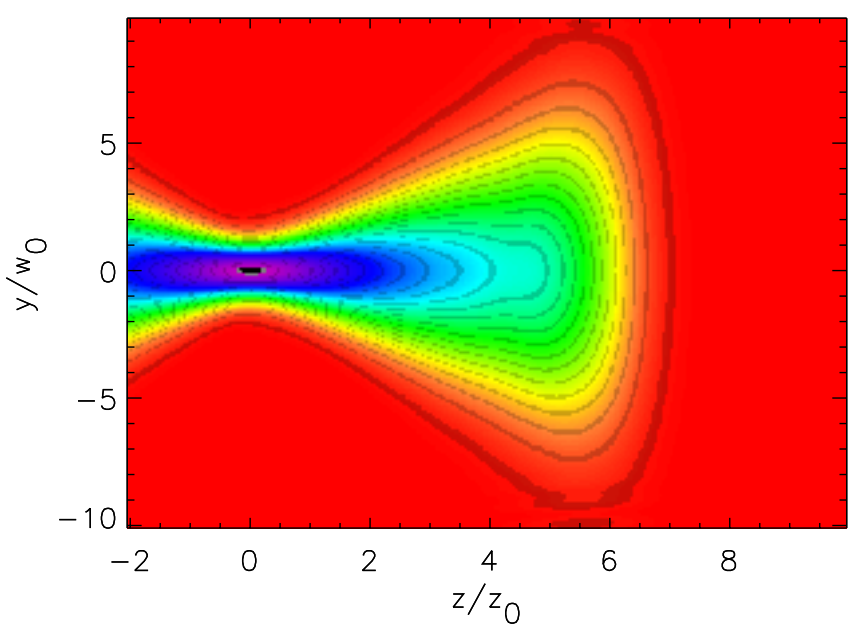

FIG. 2. (Color) Amplitude contour plot of a laser pulse launched into a vacuum domain, propagating through the focus.

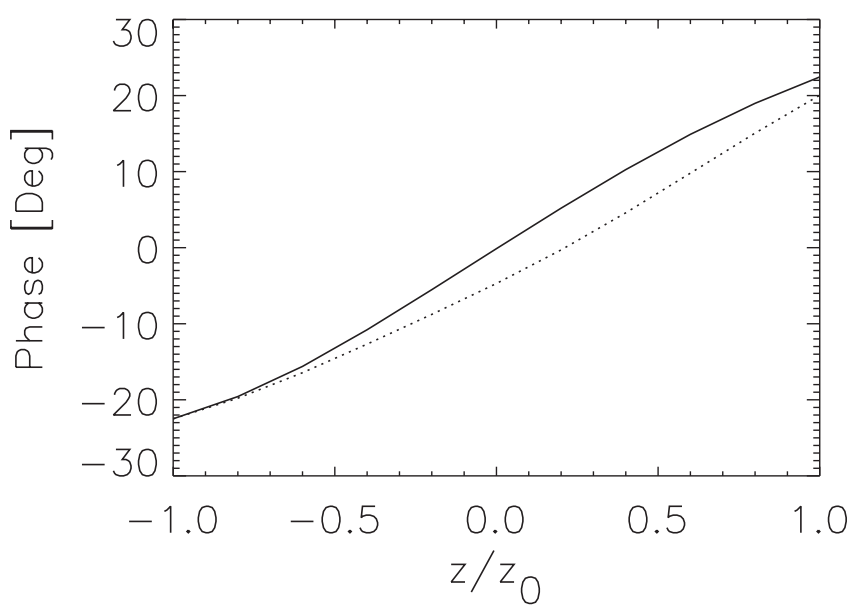

FIG. 3. Envelope phase during propagation through the focus for transverse resolutions of the laser pulse of $w_{0}=4 \Delta y$ (solid) and $w_{0}=2 \Delta y$ (dotted). The Gouy phase shift is $45^{\circ}$.

A measure for the accuracy of the envelope propagation is the phase shift experienced by the laser pulse propagating from $z=-z_{0}$ to $+z_{0}$. Figure 3 compares the phase shift observed in the simulations with two different transverse resolutions. The simulation parameters are identical to the previous example. For a transverse resolution of $w_{0}=4 \Delta y$, good agreement with the theoretical Gouy phase shift (see e.g. [17]) can be found. At a resolution of $w_{0}=2 \Delta y$, a phase error of $5 \%$ can be observed.

Scaling of the Gouy phase shift helps to determine the scaling of the field solver error. Figure 4 shows the scaling of the phase error as a function of transverse resolution. The error of a Crank-Nicholson scheme should scale at least with order $\Delta y^{2}$, which is in agreement with the $\Delta y^{3}$ relation observed here.

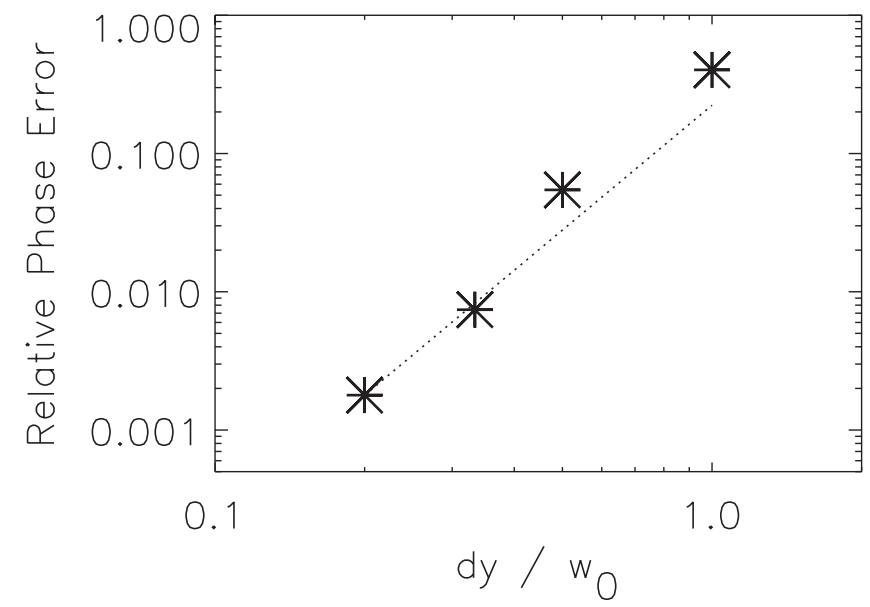

FIG. 4. Phase error of the Gouy phase shift, as a function of transverse resolution. The error follows a $\Delta y^{3}$ dependency (dotted line). 


\section{B. Comparison with PIC}

The tests in the previous section demonstrated correct envelope propagation in vacuum. The next step is now to ensure the correct interaction of the envelope model with the ponderomotive PIC model.

For the first comparison, a laser pulse of normalized laser amplitude $a_{0}=1$ is injected into a plasma of density $n=1.74 \times 10^{25} \mathrm{~m}^{-3}$ with a ratio of laser frequency to plasma frequency $\omega / \omega_{p}=10$. The plasma density is ramped up linearly over a distance of $20 \mu \mathrm{m}$. The overall computational domain is $40 \times 64 \mu \mathrm{m}$. For the full PIC simulation, the domain is represented by a grid of $800 \times$ 1280 cells in order to resolve the laser wavelength. The time step of $1.12 \times 10^{-16} \mathrm{~s}$ is determined by the CourantFriedrichs-Levy (CFL) condition.

Using the envelope model, the same computational domain can be captured with a much smaller computational grid. The above simulation was performed on an $80 \times 128$ cell grid with a time step of $1.12 \times 10^{-15} \mathrm{~s}$. The grid is 100 times smaller and the time step 10 times larger than for the full PIC model. Note that the time step in the envelope model is still bound by the CFL condition, as the wake field is updated using an explicit integration of Maxwell's equations. However, due to the much coarser grid, which now no longer needs to resolve the laser wave length, a much larger time step results.

Figure 5 shows the longitudinal electric field of the plasma wake behind the laser pulse. The envelope model captures all the features of the wake quantitatively, at a considerably lower cost. For an average 5 particles per cell, the computational cost of the full PIC simulation was approximately 200 times larger than for the envelope

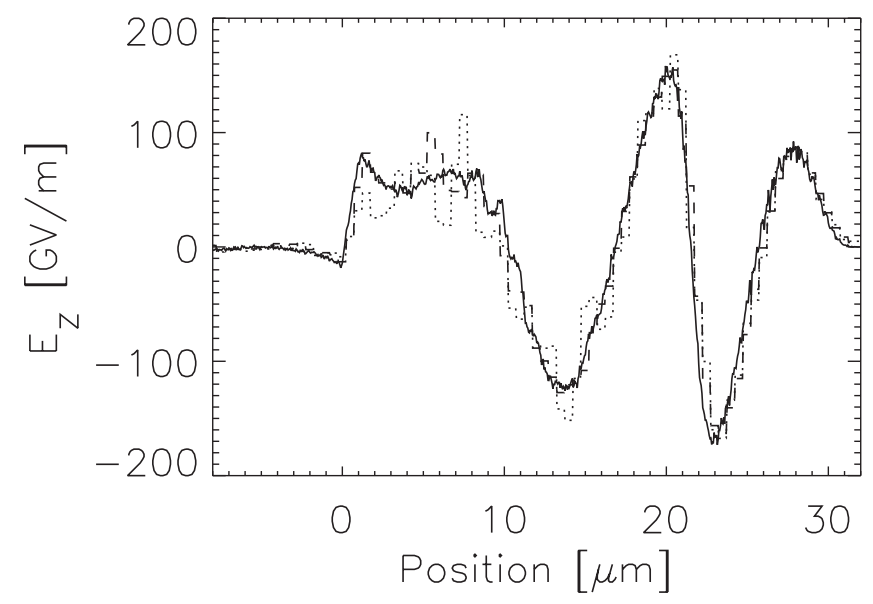

FIG. 5. Longitudinal electric field in the wake of a laser pulse, computed with full PIC (solid), as compared with the envelope model on a 100 times smaller grid and 10 times fewer particles (dashed) and with 100 times fewer particles (dotted). The plasma extends from $x=0$ to $32 \mu \mathrm{m}$, with a linear density ramp in the first $20 \mu \mathrm{m}$. model. The main difference between the full PIC simulation and the envelope model occurs at low plasma density at the onset of the plasma ramp between $x=0$ to $10 \mu \mathrm{m}$. These rapid fluctuations seen in the envelope model are due to the lower particle number and disappear with increasing particle number.

Figure 6 compares the longitudinal electron phase space between the full PIC simulation and the envelope model, for the same example problem. On the whole, the agreement is compelling. Discrepancies at lower particle density (to the left, near the foot of the density ramp) are due to the 100 fold larger weight of the envelope particles, as compared to the PIC particles. Within the laser pulse (to the right), one sees the response of the PIC particles to fast oscillations of the laser field, while the envelope particles show an averaged response to the ponderomotive force.

\section{Channel simulations}

In order to investigate the benefit of a channel to control the laser diffraction, we performed a simulation of a laser pulse propagating in a preformed channel of $0.8 \mathrm{~cm}$ length, using the newly developed envelope model. The density at the channel bottom is $1.25 \times 10^{17} \mathrm{~cm}^{-3}$, corresponding to a plasma wave length of $L=2 \pi c / \omega_{p}=95 \mu \mathrm{m}$. The channel has a parabolic profile with opening $20 \mu \mathrm{m}$. A matched $3.17 \times 10^{-13} s$ laser pulse with $\omega=2.35 \times$ $10^{15} \mathrm{~s}^{-1}$, a Rayleigh length $z_{0}=0.157 \mathrm{~cm}$ and amplitude $a_{0}=1.0$ is sent into the channel. The simulation captures a domain of $0.9 \mathrm{~cm} \times 120 \mu \mathrm{m}$, discretized on a $3000 \times 40$ cell grid.

Figure 7 shows the width of the laser pulse while it propagates through the plasma channel. For comparison, the evolution of the pulse without a channel is shown. The laser pulse is guided in the channel without significant diffraction.

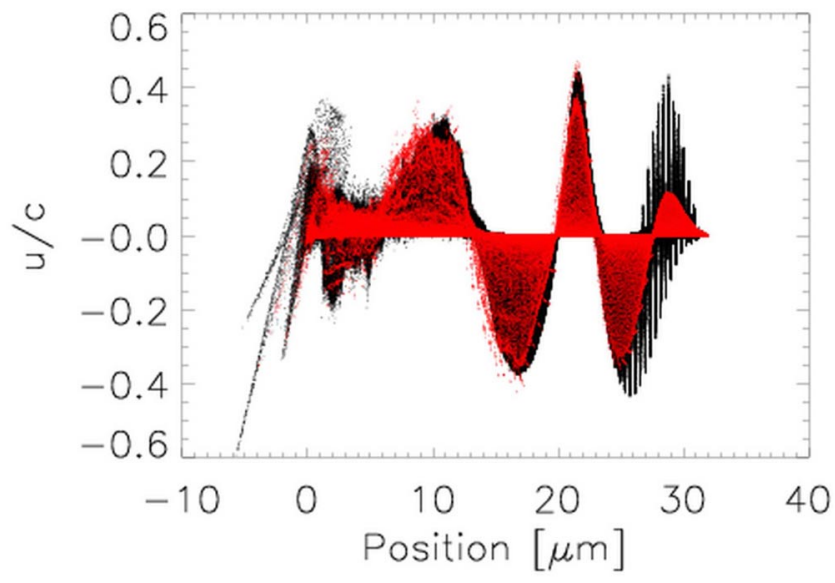

FIG. 6. (Color) Longitudinal electron phase space, computed with full PIC (black) and with the envelope model (red). 


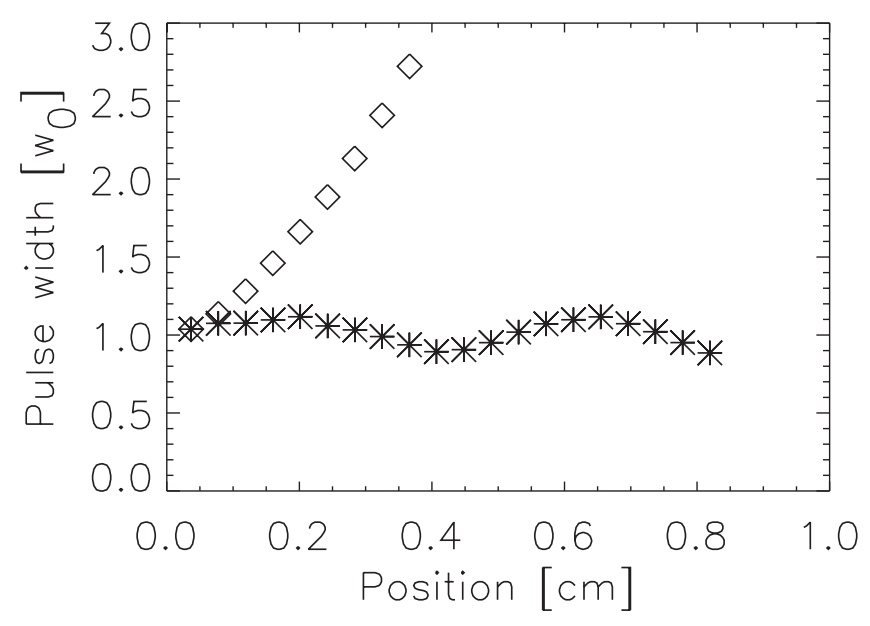

FIG. 7. Laser pulse width for propagation in a channel (stars) and without a channel (diamonds).

\section{TRANSITION RADIATION}

Transition radiation is emitted when a charged particle crosses an interface with two different dielectric constants. E.g. for a single particle crossing an interface, the energy radiated per unit frequency $d \omega$ and unit angle $d \Omega$ is given by

$$
\frac{d^{2} W}{d \omega d \Omega} \propto \frac{\beta^{2} \sin ^{2} \theta}{\left(1-\beta^{2} \cos ^{2} \theta\right)^{2}},
$$

where $\theta$ is the observation angle. The energy radiated by an electron bunch, rather than a single electron, causes radiation patterns that depend on the beam properties. Transition radiation therefore can be used as a diagnostic tool for particle beams [18]. Among other parameters, the transition radiation depends on the transverse size of the plasma. It is therefore critical to model a large enough domain to observe transition radiation in simulations. Another challenge in modeling transition radiation in underdense laser-plasma experiments is the overlap of the laser field with the THz emission. The high intensity of the laser pulse makes the transition radiation hard to observe.

Both of these problems can be overcome with the envelope model presented here. No longer requiring resolution of the laser wavelength results in high execution speed of the code and therefore enables large simulation domains. In addition, the decomposition of the electromagnetic field into the laser field and the wake field helps to evade the contrast problem.

In this section, we demonstrate the use of the envelope model for observing transition radiation. A thin preionized foil of $8 \mu \mathrm{m}$ thickness is irradiated with a strong laser pulse with $a_{0}=11$. This high laser field accelerates electrons within the foil to velocities up to $\beta=0.78$.

Figure 8 shows the electromagnetic field energy behind the foil, $30 \mathrm{fs}$ after the first particles emerge behind the foil.

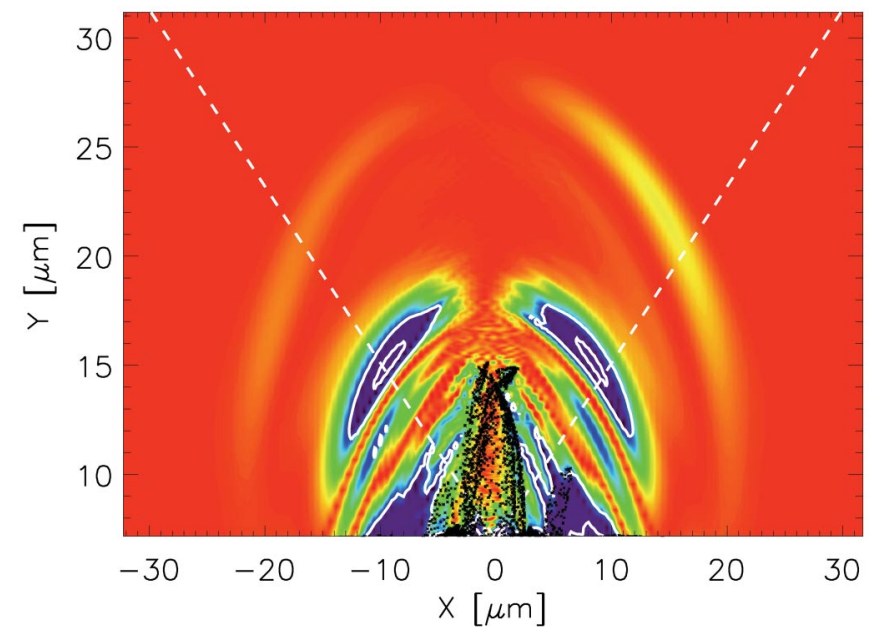

FIG. 8. (Color) Spatial distribution of the electromagnetic field energy behind the foil, 30 fs after the first particles emerge from the foil. The contours show the peak of the emitted radiation, comparing it to the expected peak emission (dashed). The black dots represent the particles emitted from the foil.

The contour lines show the peak of the radiated energy propagating at an angle of about $\theta \approx 51^{\circ}$. This angle corresponds well to the peak emission angle expected for a single particle with $\beta=0.78$, using Eq. (21).

In addition to the transition radiation due to the particle beam, another pulse can be seen that has propagated further. This pulse is due to the laser pulse transiting from the plasma into vacuum.

\section{CONCLUSION}

We have presented a ponderomotive guiding center algorithm implemented in VORPAL, that enables modeling large-scale systems for laser-plasma interaction. Especially at low plasma densities, significant saving in computing time can result. It has been demonstrated that the model can reproduce both analytical and PIC simulation results and that it can be used to model laser pulse propagation in a low density channel. In addition, we have demonstrated that the model can be used to model transition radiation. This can be helpful as a bunch length diagnostic for the electron beam, just as it leaves the plasma.

\section{ACKNOWLEDGMENTS}

The authors wish to thank Daniel Gordon, Eric Esarey, Wim Leemans, Cameron Geddes, Brad Shadwick, Carl Schroeder, Dimitre A. Dimitrov, John R. Cary, Peter Stoltz and Chet Nieter for fruitful discussions. This work was supported by the U.S. DOE Office of Science, Office of High Energy Physics under Grants No. DE-FG0204ER84097, No. DE-FC02-01ER41178, and No. DEAC02-05CH11231, including use of NERSC facilities, and by Tech-X Corporation. 
[1] C. G. R. Geddes, C. Toth, J. van Tilborg, E. Esarey, C. B. Schroeder, D. Bruhwiler, C. Nieter, J. R.Cary, and W.P. Leemans, Nature (London) 431, 538 (2004).

[2] S. P. D. Mangles et al., Nature (London), 431, 535 (2004).

[3] J. Faure et al., Nature (London), 431, 541 (2004).

[4] E. Esarey et al., IEEE Trans. Plasma Sci. 24, 252 (1996)

[5] E. Esarey and W.P. Leemans, in Proceedings of the Particle Accelerator. Conference, New York, 1999, (IEEE, Piscataway, NJ, 1999), p. 3699.

[6] D. J. Spence, A. Butler, and S. M. Hooker, J. Opt. Soc. Am. B 20, 138 (2003).

[7] D.F. Gordon, W. B. Mori, and T. M. Antonsen, IEEE Trans. Plasma Sci. 28, 1224 (2000).

[8] P. Mora and T. M. Antonsen, Phys. Plasmas 4, 217 (1997).

[9] C. Nieter and J. R. Cary, J. Comput. Phys. 196, 448 (2004).

[10] J. Krall, A. Ting, E. Esarey, and P. Sprangle, Phys. Rev. E 48, 2157 (1993).
[11] P. Sprangle et al., Phys. Rev. E 63, 056405 (2001).

[12] G. P. Agrawal, Nonlinear Fiber Optics (Academic Press, San Diego, 1995).

[13] J. Villasenor and O. Buneman, Comput. Phys. Commun. 69, 306 (1992).

[14] J. Boris, in Proceedings of the Fourth Conference on Numerical Simulation of Plasmas, edited by J.P. Boris and R.A. Shanny (Naval Research Laboratory, Washington, DC, 1970), pp. 367.

[15] R. S. Tuminaro, M. Heroux, S. A. Hutchinson, and J. N. Shadid, Technical Report No. SAND99-8801J, 1999.

[16] P. Messmer and D. L. Bruhwiler, Comput. Phys. Commun. 164, 118 (2004).

[17] E. A. Saleh and M. C. Teich, Fundamentals of Photonics (Wiley, New York, 1991).

[18] C. B. Schroeder, E. Esarey, J. van Tilborg, and W. P. Leemans, Phys. Rev. E 69, 016501 (2004). 